\title{
On Pythagorean triplet semigroups
}

\author{
Antoine Mhanna \\ SRS College Kleiat Lebanon \\ e-mail: tmhanat@yahoo.com
}

Received: 27 November 2019 Revised: 3 November $2020 \quad$ Accepted: 9 November 2020

\begin{abstract}
In this note we explain the two pseudo-Frobenius numbers for $\left\langle m^{2}-n^{2}, m^{2}+n^{2}, 2 m n\right\rangle$ where $m$ and $n$ are two coprime numbers of different parity. This lets us give an Apéry set for these numerical semigroups.
\end{abstract}

Keywords: Numerical semigroups, Primitive Pythagorean triplets, Pseudo-Frobenius number. 2010 Mathematics Subject Classification: 11D07, 11D45, 11D85, $20 \mathrm{M} 14$.

\section{Introduction and preliminaries}

Let $a_{1}, \ldots, a_{n}$ be $n$ positive integers with $\operatorname{gcd}\left(a_{1}, \ldots, a_{n}\right)=1$, the set

$$
S:=\left\{\sum_{i=1}^{s} \lambda_{i} a_{i} \mid s \in \mathbb{N}, \lambda_{i} \geq 0, \text { for all } i\right\}
$$

be called the numerical semigroup $S$ and the integers $a_{1}, \ldots, a_{n}$ be its generators. A numerical semigroup is minimally generated by $a_{1}, \ldots, a_{n}$ if we cannot remove a generator without changing the set $S$; in this case we denote $S$ by $\left\langle a_{1}, a_{2}, \ldots, a_{n}\right\rangle$. Given $S \neq \mathbb{N}$, the number $F(S):=\max \{n \in \mathbb{N} \mid n \notin S\}$ (which exists, see [5, Theorem 1.0.1]) is the Frobenius number of $S$. For a numerical semigroup $S$ let

$$
T(S):=\{x \in \mathbb{N} \mid x \notin S, x+s \in S \text {, for all } s \in S, s>0\} .
$$

The cardinality of $T(S)$ is called the type of $S$ and a number in $T(S)$ is called a pseudo-Frobenius number. The Apéry set of $S$ with respect to $n \in S$ is the set $\operatorname{Ap}(S, n)=\{s \in S \mid s-n \notin S\}$ and the genus of $S$ denoted $g(S)$ is the cardinality of $\{\mathbb{N} \backslash S\}$. 
Definition 1. A numerical semigroup is said to be Arf if for all $s, r, t \in S$ with $s \geq r \geq t$, $s+r-t \in S$. For $S=\left\langle a_{1}, \ldots, a_{n}\right\rangle$ we define for every $i \in\{2, \ldots, n\}$ :

$$
c_{i}=\min \left\{k \in \mathbb{N} \backslash\{0\} \mid k \cdot a_{i} \in\left\langle a_{1}, \ldots, a_{i-1}\right\rangle\right\},
$$

$S$ is then free if $a_{1}=c_{2} \cdots c_{n}$.

Remark $1([2,4,8])$. Let $a_{1}, a_{2}, \ldots, a_{k}$ be positive integers. If $\operatorname{gcd}\left(a_{2}, \ldots, a_{n}\right)=d$ and $a_{j}=d \cdot a_{j}^{\prime}$ for each $j>1$, then

- The type of $\left\langle a_{1}, a_{2}, \ldots, a_{n}\right\rangle$ equals the type of $\left\langle a_{1}, a_{2}^{\prime}, \ldots, a_{n}^{\prime}\right\rangle$.

- The type of $S:=\left\langle a_{1}, a_{2}, a_{3}\right\rangle$ is at most two (see [2, Theorem 11]) and it equals two if $S$ has pairwise coprime minimal generators (see [7]).

- $\operatorname{Ap}(S, n)$ has n elements and $g(S)=\frac{1}{n} \sum_{w \in \operatorname{Ap}(S, n)} w-\frac{n-1}{2}$ (see [8, Chapter 1]).

A survey on finding Frobenius numbers for numerical semigroups can be found in [5].

A Pythagorean triplet is a positive integer triplet $(x, y, z)$ verifying $x^{2}+y^{2}=z^{2}$. We say that this triplet is primitive if any two integers from $x, y, z$ are coprime and we have: Every primitive Pythagorean triplet can be expressed as $\left(m^{2}-n^{2}, 2 m n, m^{2}+n^{2}\right)$ where $m$ and $n$ are coprime numbers of different parity.

Proposition 1. Let $a$ and $b$ be two coprime positive integers and let $\left(x_{0}, y_{0}\right)$ denote the nonnegative couple (when it exists) verifying $a x_{0}+b y_{0}=n, 0 \leq y_{0}<a,\left(b y_{0}=n(\bmod a)\right)$ then the number of nonnegative integer solutions to the equation $a x+b y=n$ equals $\left\lfloor\frac{n-b y_{0}}{a b}\right\rfloor+1$.

Proof. Set $x_{i}=x_{0}-i b$ and $y_{i}=i a+y_{0}$, since $a$ and $b$ are coprime, for every $i$ there is a unique $y_{i}$ with $i a \leq y_{i}<(i+1) a$ such that $a x_{i}+b y_{i}=n$. Counting the nonnegative couples $\left(x_{i}, y_{i}\right)$ we get the result.

Corollary 1. Let $m$ and $n$ be two coprime positive integers of different parity. If $t \in \mathbb{N}, t \geq 1$ and $m>(t+1) n$, then $(t n, t n)$ is the unique nonnegative solution to

$$
2 t m n=(m-n) x+y(m+n) .
$$

Proof. We apply Proposition $1 y_{0}=t n<m-n$, notice that $t m n-t n^{2}<m^{2}-n^{2} \Longleftrightarrow$ $t m(n-m)-n^{2}(t-1)<0$.

Corollary 2 (Bézout). The integer solutions of (1) are of the form:

$$
(t n+k(m+n), t n-k(m-n))
$$

for some $k \in \mathbb{Z}$. 


\section{Main result}

From the definition of a pseudo-Frobenius number $F$ for a given $S:=\left\langle a_{1}, a_{2}, a_{3}\right\rangle, z:=F+a_{3} \in S$ but since $F \notin S, z=\sum_{i=1}^{2} u_{i} a_{i}$, consequently any such number $F$ can be written as $u a_{1}+v a_{2}-a_{3}$ for some $u \geq 0$ and $v \geq 0$. It is known ([6]) that for any numerical semigroup $\langle a, b\rangle$ a positive integer $x \notin S$ if and only if $x=\alpha a-\beta b$ for some $0<\alpha<b$ and $0<\beta<a$.

We set $a_{1}=2 m n, a_{2}=m^{2}+n^{2}$ and $a_{3}=m^{2}-n^{2}$ so $S:=\left\langle m^{2}-n^{2}, m^{2}+n^{2}, 2 m n\right\rangle$ : when $m=n+1, a_{1}=2 n(n+1), a_{2}=2 n^{2}+2 n+1=(2 n+1)(2 n+1)-2 n(n+1)=$ $2 n(n+1)(2 n)-\left(2 n^{2}-1\right)(2 n+1)$ and $a_{3}=2 n+1$, using Theorem 11's proof [2], we can find the two pseudo-Frobenius numbers of this semigroup (we leave it as an exercise). This method does not easily settle the general case, however the Frobenius number $F(S)$ (as $g(S)$ ) was given in Example 3 of [1], see also [3]. Recently a complete (different) study of Pythagorean semigroups including finding $T(S), F(S)$ and $g(S)$ was done by A. Tripathi and E. F. Elizeche [9]. We thank the authors for correspondences.

Remark 2. We have $m(2 m n)=n\left(m^{2}-n^{2}\right)+n\left(m^{2}+n^{2}\right), m\left(m^{2}+n^{2}\right)=n(2 m n)+m\left(m^{2}-n^{2}\right)$ and $(m+n)\left(m^{2}-n^{2}\right)=(m-n)\left(m^{2}+n^{2}\right)+(m-n)(2 m n)$.

Theorem 2.1. Let $S=\left\langle m^{2}-n^{2}, m^{2}+n^{2}, 2 m n\right\rangle$, $m$ coprime with $n$ and of distinct parity, then $T(S)=\{P F(S), F(S)\}$ where

$$
P F(S)=(m-1)\left(m^{2}+n^{2}\right)+(n-1)\left(m^{2}-n^{2}\right)-2 m n
$$

and

$$
F(S)=(m-1)\left(m^{2}-n^{2}\right)+(m-1) 2 m n-\left(m^{2}+n^{2}\right)
$$

Proof. The proof is straight computationally, we verify that the two given numbers can not be in $S$ and that $T(S)+a_{i} \in S,(i=1,2,3)$.

$$
\begin{aligned}
& F(S)+a_{2}=(m-1)\left(m^{2}-n^{2}\right)+(m-1) 2 m n \\
& F(S)+a_{3}=(m-1)\left(m^{2}+n^{2}\right)+(m-n-1) 2 m n \\
& F(S)+a_{1}=(n-1)\left(m^{2}+n^{2}\right)+(m+n-1)\left(m^{2}-n^{2}\right) \\
& P F(S)+a_{3}=(m-n-1)\left(m^{2}+n^{2}\right)+(m-1) 2 m n \\
& P F(S)+a_{1}=(m-1)\left(m^{2}+n^{2}\right)+(n-1)\left(m^{2}-n^{2}\right) \\
& P F(S)+a_{2}=(n-1) 2 m n+(m+n-1)\left(m^{2}-n^{2}\right)
\end{aligned}
$$

Suppose $F(S)=\alpha\left(m^{2}+n^{2}\right)+\beta\left(m^{2}-n^{2}\right)+\gamma(2 m n)$ where $\alpha, \beta, \gamma$ are nonnegative and we can assume that $\gamma<m$ by Remark 2 with $\alpha<2 m-3$. If $\gamma=\alpha+v \geq \alpha$, then $F(S)=$ $\alpha(m+n)^{2}+\beta(m-n)(m+n)+v(2 m n)$ implying that $(m+n)$ divides $2 m n(v-m)$, a contradiction. If otherwise $\gamma<\alpha=\gamma+v<2 m-3$, we get $F(S)=\gamma(m+n)^{2}+\beta(m-n)(m+n)+v\left(m^{2}+n^{2}\right)$, which implies that $(m+n)$ divides $2 m n(v+m)$, so $v=n$ or $v=m+2 n$. In case $v=n$, respectively $v=m+2 n$, after simplifying by $(m+n)$, we need to solve $2 m n=(\gamma+1+n)(m+n)+$ $(\beta-m+1)(m-n)$, respectively, $4 m n=(\gamma+1+m+2 n)(m+n)+(\beta-m+1)(m-n)$, from 
Corollary 2 we see that supposing $n+1+\gamma=n+k(m-n),(k \geq 1)($ so $\beta-m+1=n-k(m+n))$, $\beta=m-1+n-k(m+n)<0$, a contradiction. The same contradiction is true for the respective case.

For the other number $\operatorname{PF}(S)=(m-1)\left(m^{2}+n^{2}\right)+(n-1)\left(m^{2}-n^{2}\right)-2 m n$ the same arguments hold: Suppose $P F(S)=\alpha\left(m^{2}+n^{2}\right)+\beta\left(m^{2}-n^{2}\right)+\gamma(2 m n)$ where $\alpha, \beta, \gamma$ are nonnegative and we can assume that $\gamma<m$ by Remark 2 with $\alpha<m+2 n-3$. If $\gamma \leq \alpha=\gamma+v$, then $P F(S)=\gamma(m+n)^{2}+\beta(m-n)(m+n)+v\left(m^{2}+n^{2}+2 m n-2 m n\right)$ implying that $(m+n)$ divides $2 m n(v-m)$, so $v$ must equal $m$, simplifying by $(m+n)$ we have to solve $(\gamma+1)(m+n)=(n-1-\beta)(m-n)$, a contradiction. If otherwise $\alpha<\gamma=\alpha+v<m$, we get $P F(S)=\alpha(m+n)^{2}+\beta(m-n)(m+n)+v(2 m n)$, which implies that $(m+n)$ divides $2 m n(v+m)$, so $v=n$. After simplifying by $(m+n)$, we need to solve $2 m n=(m-\alpha-1)(m+n)+(n-1-\beta)(m-n)$ from Corollary 2 we see that $\alpha$ and $\beta$ cannot be both nonnegative, a contradiction.

Now from Remark 1 and Theorem 2.1 we can give the Apéry set for $\left\langle m^{2}-n^{2}, m^{2}+n^{2}, 2 m n\right\rangle$.

Lemma 2.2. Let $S=\left\langle m^{2}-n^{2}, m^{2}+n^{2}, 2 m n\right\rangle$, then $\operatorname{Ap}(S, 2 m n)=\left\{a\left(m^{2}+n^{2}\right)+b\left(m^{2}-n^{2}\right)\right.$, $0 \leq a \leq(m-1)$ and $0 \leq b \leq(n-1)$ or $0 \leq a \leq n-1$ and $n \leq b \leq m+n-1\}$ and

$$
g(S)=\frac{m^{3}-n^{3}+1}{2}+m^{2} n-m^{2}-m n
$$

A numerical semigroup $S$ is symmetric, respectively pseudo-symmetric, if $T(S)=\{F(S)\}$, respectively $T(S)=\left\{F(S), \frac{F(S)}{2}\right\}$. For $\left\langle m^{2}-n^{2}, m^{2}+n^{2}, 2 m n\right\rangle$

$$
2 \cdot P F(S)-F(S)>(m-3) \cdot\left(m^{2}+n^{2}\right),
$$

by Theorem 2.1's expressions, a Pythagorean triplet semigroup is not free nor symmetric and it is Arf and pseudo-symmetric if $m=2=n+1$.

\section{Acknowledgements}

Thanks to reviewers for constructive advice.

\section{References}

[1] Fel, L. G. (2006). Frobenius Problem for Semigroups $S\left(d_{1}, d_{2}, d_{3}\right)$, Funct. Anal. Other Math., 1, 119-157.

[2] Fröberg, R., Gottlieb, C., \& Hägkvist, R. (1987). On numerical semigroups, Semigroup Forum, 35, 63-83.

[3] Gil, B. K., Han, J.-W., Kim, T. H., Koo, R. H., Lee, B. W., Lee, J., Nam, K. S., Park, H. W., \& Park P.-S. (2015). Frobenius numbers of Pythagorean triples, Int. J. Number Theory, 11, 613-619. 
[4] Johnson, S. M. (1960). A Linear Diophantine Problem, Can. J. Math., 12(2), 390-398.

[5] Ramírez Alfonsín, J. L. (2005). The Diophantine Frobenius Problem, Oxford Univ. Press, Oxford.

[6] Rosales, J. C., \& Branco, M. B. (2011). The Frobenius problem for numerical semigroups, Journal of Number Theory, 131, 2310-2319.

[7] Rosales, J. C., \& García-Sánchez, P. A. (2004). Numerical semigroups with embedding dimension three, Archiv der Mathematik, 83, 488-496.

[8] Rosales, J. C., \& García-Sánchez, P. A. (2009). Numerical semigroups, Springer, New York.

[9] Tripathi, A., \& Elizeche, E. F. (2020). On numerical semigroups generated by primitive Pythagorean triplets, Integers, 20, A75. 